\title{
A Quantitative Method for Evaluating Reconstructed One-Dimensional Bifurcation Diagrams
}

\author{
Yoshitaka Itoh, Masaharu Adachi* \\ Department of Electrical and Electronic Engineering, Tokyo Denki University, 5 Senju-Asachicho Adachi-ku, \\ Tokyo , Japan. \\ * Corresponding author. Tel.: +81-3-5284-5407; email: adachi[at-mark]eee.dendai.ac.jp \\ Manuscript submitted March 10, 2017; accepted June 8, 2017. \\ doi: $10.17706 /$ jcp.13.3.271-278
}

\begin{abstract}
We describe a quantitative method for evaluating reconstructed 1-dimensional bifurcation diagrams. We estimate the oscillatory patterns of time-series data by reconstructing the bifurcation diagrams from time-series data alone. Such reconstruction can be used for real-world systems that have variable parameters, such as electric current and power, temperature, pressure, and concentration. In the conventional method, the reconstructed bifurcation diagram is qualitatively compared with the original one. Here, we evaluate the reconstructed 1-dimensional bifurcation diagrams by means of quantitative comparison with the original one. We also present the results of numerical experiments, demonstrating that our method is useful for quantitative evaluation of reconstructed bifurcation diagrams for the Hénon map and the Rössler equations.
\end{abstract}

Key words: Chaos, reconstruction of bifurcation diagram, time-series prediction, extreme learning machine, evaluation methods, levenshtein distance.

\section{Introduction}

A bifurcation diagram (BD) is a visual summary of the succession for time-series data that are generated by a dynamical system as its parameters are varied. The oscillatory pattern of time-series data sometimes changes with a parameter. Here, the oscillatory patterns are periodic, quasi-periodic, and chaotic, and we estimate them by reconstructing the BDs. Such reconstruction could be used for real-world systems that have variable parameters, such as electric current and power, temperature, pressure, and concentration.

Tokunaga et al. proposed a method for reconstructing BDs from time-series data alone [1]. This method uses some of the time-series data that are generated by one dynamical system with some parameters. In addition, this method assumes that each parameter of the time-series data is close to the others. Moreover, it is better that these time-series show various oscillatory patterns. After it was first proposed, the method was studied further by several research groups [2]-[6].

Tokunaga et al. used three-layer neural networks with back propagation (BP) learning as a time-series predictor. Reconstructing a BD requires the data of each time series to be learned repeatedly, and so the computational cost is very high. Y. Tada and M. Adachi proposed using an extreme learning machine (ELM) as a predictor for these types of problems [7]. ELMs are suited for reconstruction of BDs because not only is their computational cost lower but also the accuracy is higher for prediction of single time-series data than when using BP learning. In [7], they show the results of reconstruction using ELM for the Rössler equations, which has two parameters. In addition, they quantitatively evaluate the reconstruction accuracy for 
two-dimensional BDs. We have previously proposed a method for estimating the Lyapunov spectra of reconstructed BDs [8]. We propose a method for quantitative evaluation of the reconstructed 1-dimensional BDs.

The rest of this paper is organized as follows. In Section II, we explain the reconstruction method applied to BDs. In Section III, we describe a method for evaluating the reconstructed 1-dimensional BDs. In Section IV, we present the results of our numerical experiments. Finally, we give conclusions in Section V.

\section{Procedure for Paper Submission}

In this section, we describe the method for reconstructing a BD [1] using only some time-series data sets that are generated by one dynamical system with some parameters. Here, the time-series data set $s_{n} \in \mathbb{R}^{U}$ is to be generated with parameter $p_{n} \in \mathbb{R}^{A}$, where $U$ is the length of time-series data required for training and $A$ is the dimensionality of the parameters. Therefore, time-series data sets $s_{1}, s_{2}, \cdots, s_{N}$ correspond to parameter sets $\mathrm{p}_{1}, \mathrm{p}_{2}, \cdots, \mathrm{p}_{\mathrm{N}}$, respectively, where $\mathrm{N}$ is the number of time-series data sets. The algorithm is as follows.

1. We make a time-series predictor for each time-series data set $s_{n}$ :

$$
y(t+1)=P\left(w_{n}, y(t)\right)
$$

where

$$
\begin{gathered}
\boldsymbol{y}(t)=[y(t) y(t-k) \cdots y(t-(Y-1) k)]^{T}, \\
\boldsymbol{y}(t+1)=[y(t+1) y(t-k+1) \cdots y(t-(Y-1) k+1)]^{T} .
\end{gathered}
$$

Here, $\mathrm{P}(\cdot)$ is the time-series predictor, $w_{n} \in \mathbb{R}^{W}$ is the set of trained connection weights for the time-series data sets $s_{n}, \mathrm{k}$ is the delay time, and $y(t) \in \mathbb{R}^{Y}$ and $y(t+1) \in \mathbb{R}^{Y}$ are the input and the output, respectively, of the time-series predictor. Terms $Y$ and $W$ are the input and output dimensionalities of the time-series predictor and the number of trained connection weights, respectively. In this paper, the dimensionality of output neurons is set to that of the input neurons in order to calculate the Jacobian matrix of the time-series predictor, which is used for estimating the Lyapunov exponents. In this paper, the dimensionality $Y$ of the input and output neurons is set to that of the target dynamical system. We use an ELM as the time-series predictor. This is a feedforward neural network with a single hidden layer; the structure is shown in Fig. 1. The ELM is trained on only the connection weights of output neurons. The connection weights and biases of hidden neurons are initially generated as random values and then fixed.

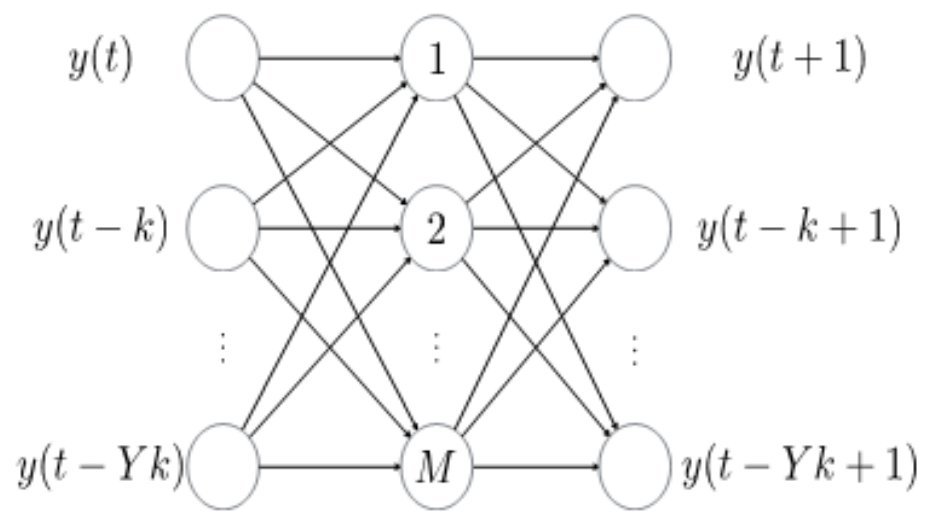

Fig. 1. Structure of the ELM. 
2. We apply principal component analysis (PCA) to the trained connection weights $w_{n}$ of the time-series predictor. Eigenvectors $u \in \mathbb{R}^{W}$, eigenvalues $\lambda_{i} \in \mathbb{R}(i=1, \cdots, W)$, estimated dimensionality $Q$, and principal component coefficients $\gamma \in \mathbb{R}^{Q}$ are obtained from the PCA. Here, we assume that $Q$ is defined such that the $Q$ th cumulative contribution ratio is the first to exceed $80 \%$.

3. We reconstruct the BD by using the result of the PCA. A set of new connection weights is obtained:

$$
\widetilde{\boldsymbol{w}}=\left[\begin{array}{llll}
\boldsymbol{u}_{1} & \boldsymbol{u}_{2} & \cdots & \boldsymbol{u}_{Q}
\end{array}\right] \gamma+\overline{\boldsymbol{w}}
$$

where $\bar{w} \in \mathbb{R}^{W}$ is the average of the trained connection weights and $\gamma$ is used as the parameter for reconstructing the $\mathrm{BD}$. The $\mathrm{BD}$ is reconstructed by repeatedly generating time-series data from new time-series predictors. Specifically,

$$
\boldsymbol{y}(t+1)=P(\widetilde{\boldsymbol{w}}, \boldsymbol{y}(t))
$$

where the new connection weights $\widetilde{w}$ are obtained for each parameter $\gamma$ in the principal component space.

4. We estimate the Lyapunov exponents of the reconstructed BD. Here, the Lyapunov exponent of each time-series predictor is estimated by using the methods of [9] and [10]. First, we calculate the Jacobian matrix of the time-series predictor $\mathrm{P}(\cdot)$ in (3) by using the method of [11]. Next, we decompose the Jacobian matrix by QR decomposition. Then, we obtain the Lyapunov exponents from

$$
\mu_{j}=\lim _{\phi \rightarrow \infty} \frac{1}{\phi} \sum_{t=1}^{\phi} \log r_{j j}(t)(j=1, \cdots, Y)
$$

where $r_{j j}(\mathrm{t})$ is the jth diagonal component of a $Y \times Y$ real upper triangular matrix $R(t)$ obtained by QR decomposition and $\phi$ is the number of iterations.

\section{Evaluating Reconstructed 1-Dimensinal BDs}

The proposed evaluation method is focused on the bifurcation structure. It compares the transition route from periodic to chaotic solutions, and the cycle number in windows. The BD is reconstructed by being expanded in some intervals and contracted in others. Therefore, this evaluation method does not consider the size of the cycle solution region or that of the chaotic region.

\subsection{Evaluation}

The proposed method compares the sequential order of the cycle numbers between the original and reconstructed BDs. First, we obtain the cycle number at the local minima of Lyapunov exponents in each section for which the interval contains a point whose Lyapunov exponents becomes zero and a next point whose Lyapunov exponent becomes zero. This is done because the time-series data are unstable at points near a period-doubling bifurcation and stable at points around the local minima of Lyapunov exponents. Notably, the Lyapunov exponent becomes zero at the points where period-doubling bifurcation occurs. Moreover, we compare not only the cycle number but also the chaotic regions. Therefore, we take the starting point of the analysis of a chaotic region to be one at which the Lyapunov exponent becomes greater than zero. We use the Levenshtein distance to compare the chaotic regions and the sequential order of the 
cycle numbers in the reconstructed BD with those in the original BD.

\subsection{Levenshtein Distance}

The Levenshtein distance [12] is a method for measuring the distance between two words. The distance between two words is defined as the minimum edit count required to change one word into the other, where edits are an insertion, deletion, or substitution. The algorithm is as follows.

- Set $\mathrm{O}$ and $\mathrm{H}$ to be the cycle numbers for the original and the reconstructed BD, respectively. We define $f=\left[f_{1} f_{2} \cdots f_{O}\right]$ and $g=\left[g_{1} g_{2} \cdots g_{H}\right]$ to be the sequential orders of cycle numbers in the original and reconstructed BDs, respectively.

- Construct a matrix $\Upsilon \in \mathbb{R}^{(0+1) \times(H+1)}$.

- Examine a cost $C_{o h}$ for every combination of $f_{o}$ from $f$ and $g_{h}$ from $g$. If $f_{o}=g_{h}$, then $C_{o h}=0$. If $\mathrm{f}_{\mathrm{o}} \neq \mathrm{g}_{\mathrm{h}}$, then $\mathrm{C}_{\mathrm{oh}}=1$.

- Set cell $v[\mathrm{o}, \mathrm{h}]$ of the matrix $\Upsilon$ to the minimum value among the following:

- the cell immediately above plus one: $v[0-1, h]+1$,

- the cell immediately to the left plus one: $v[0, h-1]+1$,

- the cell diagonally above and to the left plus the cost: $v[o-1, h-1]+C_{o h}$.

- Once steps iii and iv are complete, the Levenshtein distance is obtained from cell $v[0+1, H+1]$.

In this paper, the Levenshtein distance compares the chaotic regions and the sequential orders of the cycle numbers between the original and reconstructed BDs. Here, insertions, deletions, and substitutions refer to shortage, excess, and failure, respectively, of the cycle number or chaos in the reconstructed BDs.

\section{Simulation Experiments}

We compare the Levenshtein distances of the reconstructed BDs of the Hénon map and Rössler equations with those of the original ones. For this experiment, we reconstruct the BDs from 5-, 7-, and 9-tuples of time-series data.

\subsection{Experimental Conditions for the Hénon Map}

The Hénon map is given by

$$
\begin{gathered}
\chi(t+1)=\psi(t)+1-\alpha \chi^{2}(t), \\
\psi(t+1)=\beta \chi(t),
\end{gathered}
$$

where $\alpha$ and $\beta$ are parameters and time-series data of $\chi(t)$ are used for the reconstruction. These parameters are determined by

$$
\begin{gathered}
\alpha_{n}=-0.2 \cos \left(\frac{2 \pi(n-1)}{N-1}\right)+1.2, \\
\beta=0.3
\end{gathered}
$$

where $N$ is the number of bifurcation paths (i.e., $N=5,7$ or 9 in this experiment). We generate time-series data $s_{n}$ for $n=1, \cdots, N$ with $\alpha_{n}$ as the $\alpha$ of (5). We use 1,000 data from each time series $\chi(t)$ to train the time-series predictor. For the Hénon map, the numbers of input, hidden, and output neurons of the time-series predictor are 2,10 , and 2, respectively, and the delay time $k$ is set to 1 . Thus, the input and desired output relations of the time-series predictor are $\chi(t)$ and $\chi(t-1)$ for the input, and 
$\chi(t+1)$ and $\chi(t)$ for the output.

We define a range of parameters for comparison of reconstructed BDs with the structural features of the original BDs. The chosen range contains the transition route from periodic to chaotic solutions, as well as the periodic windows. For the BD of the Hénon map, we define the start point to be the period-doubling bifurcation from a 4-cycle to an 8-cycle, and the end point to be the first point at which the minimum value of the time-series data is less than -1.3. The parameter interval width $\Delta \mathrm{p} \in \mathbb{R}$ is defined by

$$
\Delta p=\min _{K \rightarrow \infty} \frac{p^{(\text {end })}-p^{(\text {start })}}{K}
$$

where $p^{\text {(start) }}$ and $p^{\text {(end) }}$ are the parameter values at the start and end points, respectively, and $K$ is the number of parameters for the BD. In this experiment, we set $K$ to 3000 .

\subsection{Result for the Hénon Map}

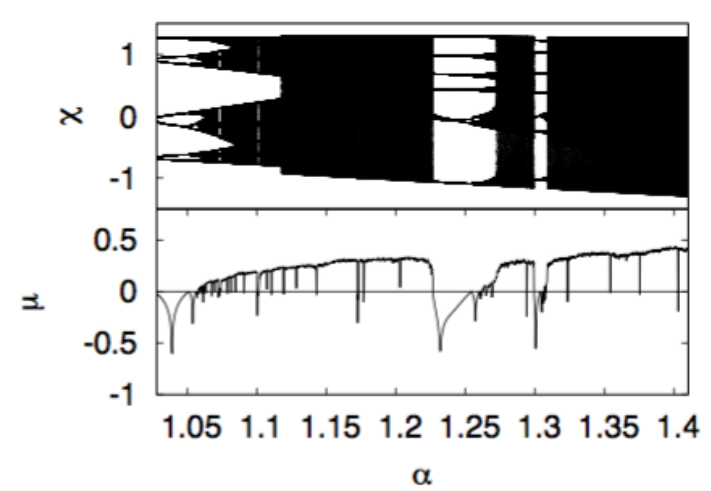

(a) Original.

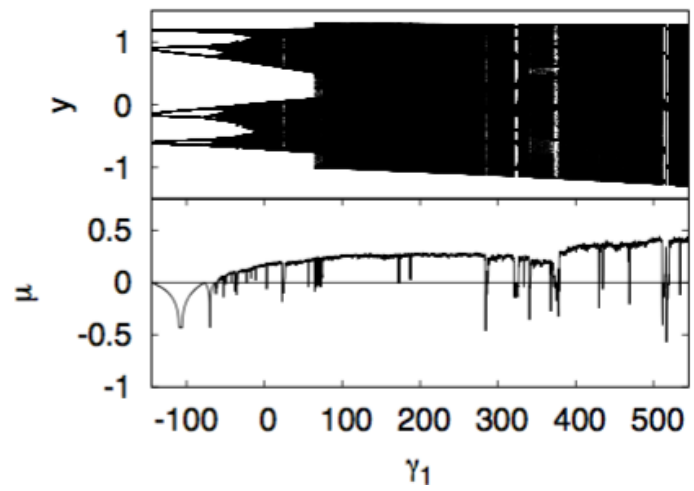

(c)7-tuple.

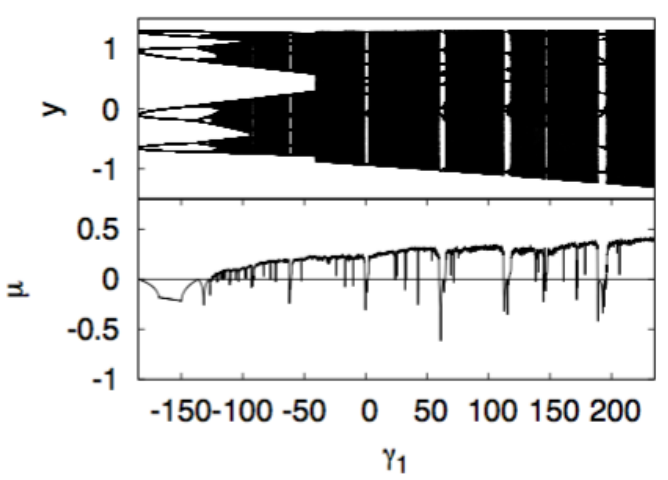

(b)5-tuple.

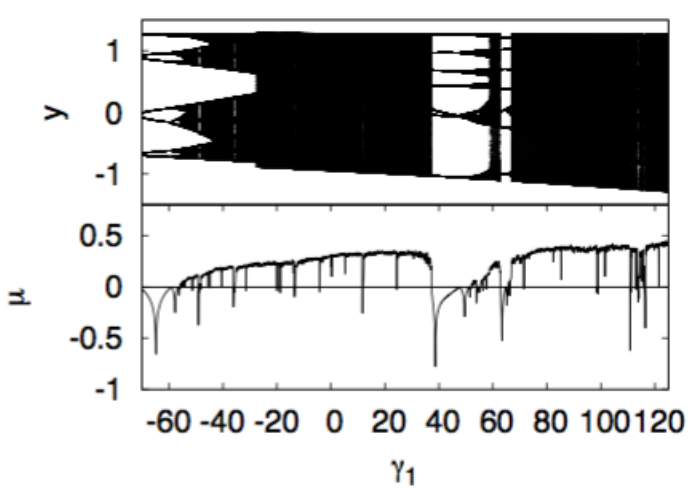

(d)9-tuple.

Fig. 2. Original and reconstructed BDs for the Hénon map.

Fig. 2 (a) shows the original BD of the Hénon map with the largest Lyapunov exponent.

Fig. 2 (b), (c), and (d) show the reconstructed BDs with the largest Lyapunov exponents that are generated from the 5-, 7-, and 9-tuple time-series data, respectively. The panels in each figure show the BD (upper) and the largest Lyapunov exponents (lower). Table 1 lists the Levenshtein distances of the reconstructed BDs in Fig. 2 (b), (c), and (d) relative to the original one in Fig. 2 (a). This shows that the more time-series data sets we use, the higher the reconstruction accuracy.

This corresponds to a qualitative comparison of Fig. 2 (b), (c), and (d) against Fig. 2 (a), in which we see that Fig. 2 (d) looks the most like Fig. 2 (a). 
Table 1. Levenshtein Distances for the Hénon Map

\begin{tabular}{ccc}
\hline \hline 5-tuple & 7-tuple & 9-tuple \\
\hline 29 & 27 & 23 \\
\hline \hline
\end{tabular}

\subsection{Experimental Conditions for the Rössler Equations}

The Rössler equations are

$$
\begin{gathered}
\frac{\mathrm{d} \xi}{\mathrm{d} \tau}=-\eta-\zeta, \\
\frac{\mathrm{d} \eta}{\mathrm{d} \tau}=\xi+v \eta, \\
\frac{\mathrm{d} \zeta}{\mathrm{d} \tau}=\epsilon \xi-(\sigma-\xi) \zeta,
\end{gathered}
$$

where $v, \epsilon$ and $\sigma$ are parameters and time-series data of $\eta$ are used for the reconstruction of the BDs. These parameters are determined by

$$
\begin{gathered}
\sigma_{\mathrm{n}}=-0.5 \cos \left(\frac{2 \pi(n-1)}{N-1}\right)+3.7, \\
u=0.33 \\
\epsilon=0.3
\end{gathered}
$$

where $N$ is the number of bifurcation paths (i.e., $N=5,7$ or 9 in this experiment). We generate time-series data $s_{n}$ for $n=1, \cdots, N$ with $\sigma_{n}$ as the $\sigma$ of (12). We do so by using a third-order Runge-Kutta method with a time step of $\Delta \tau=0.01$. We use 5,000 data from each time series to train the time-series predictor. Here, one step for the predictor corresponds to $5 \Delta \tau$ for the target system. For the Rössler equations, the numbers of input, hidden, and output neurons of the time-series predictor are 3, 50, and 3, respectively, and the delay time $k$ is set to be 16 . Thus, the input and desired output relations of the time-series predictor are $\eta(\tau), \eta(\tau-16 \times 5 \Delta \tau)$, and $\eta(\tau-32 \times 5 \Delta \tau)$ for input; $\eta(\tau-1 \times 5 \Delta \tau)$, $\eta(\tau-15 \times 5 \Delta \tau)$, and $\eta(\tau-31 \times 5 \Delta \tau)$ for output. For the Rössler equations, we use a difference time series as the desired output, applying the method proposed by [7].

For the BDs of the Rössler equations, we define the start point as the period-doubling bifurcation from periodicity 4 to periodicity 8 , and the end point as the point at which the maximum value of the time-series data is more than 4.5 .

\subsection{Results for the Rössler Equations}

Fig. 3 (a) shows the original BD with its Lyapunov spectrum. Fig. 3 (b), (c), and (d) show the reconstructed BDs with the Lyapunov spectra that are generated from the 5-, 7-, and 9-tuple time-series data, respectively. In these figures, the upper panel shows the BD, and the middle and lower panels show the largest and second-largest Lyapunov exponents, respectively. In addition, we plot the local maxima of each time-series data set in the BDs.

We list the Levenshtein distances of Fig. 3 (b), (c), and (d) from Fig. 3 (a) in Table 2. This shows that the more time-series data sets we use, the more accurately the BD is reconstructed. This corresponds to a qualitative comparison of Fig. 3 (b), (c), and (d) against Fig. 3 (a). We see that Fig. 3 (d) looks the most like Fig. 3 (a). 


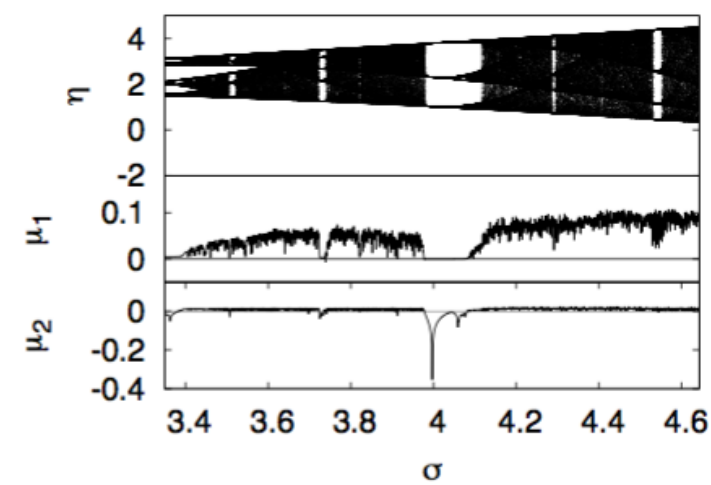

(a)Original.

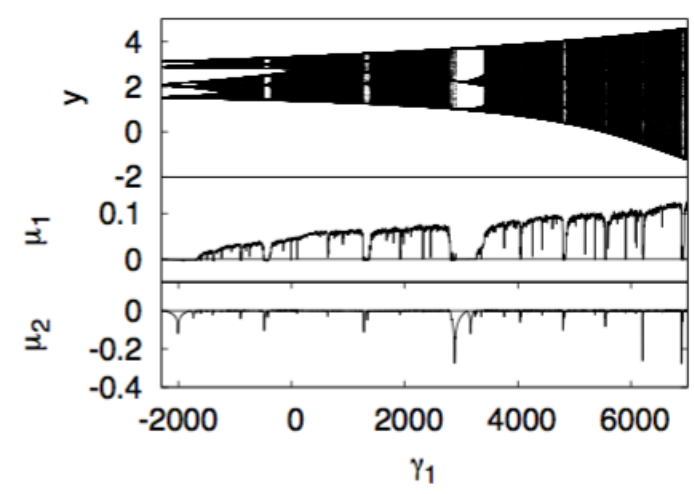

(c)7-tuple.

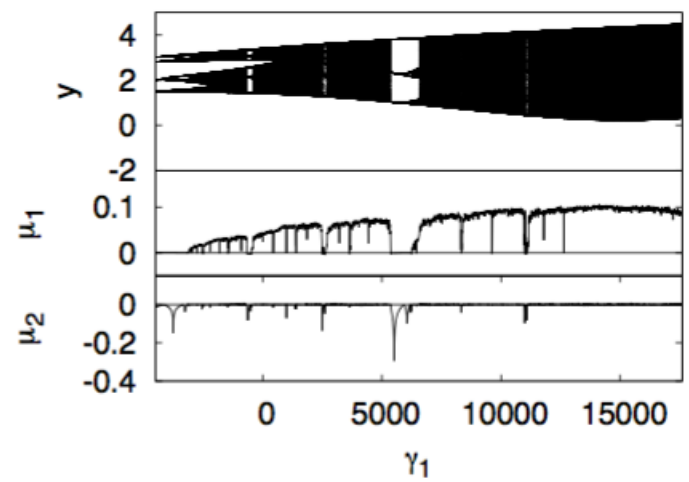

(b)5-tuple.

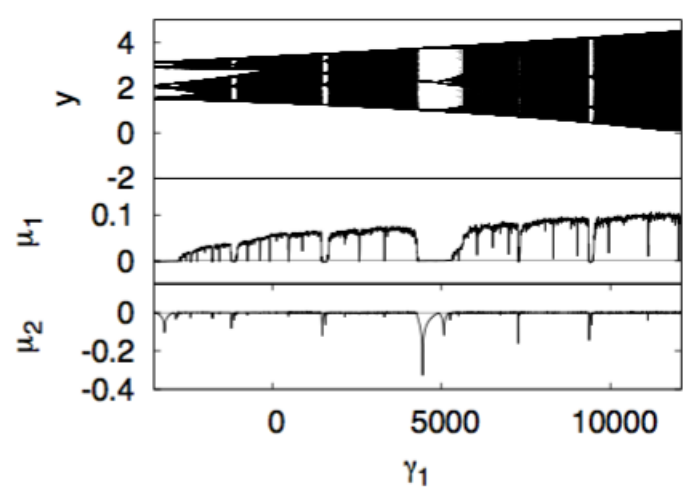

(d)9-tuple.

Fig. 3. Original and reconstructed BDs for the Rössler equations.

Table 2. Levenshtein Distances for the Rössler Map

\begin{tabular}{ccc}
\hline \hline 5-tuple & 7-tuple & 9-tuple \\
\hline 17 & 16 & 11 \\
\hline \hline
\end{tabular}

\section{Conclusions}

In this paper, we presented a method for quantitatively evaluating reconstructed 1-dimensional BDs. This method compares the chaotic regions and the sequential order of the cycle numbers between the original and reconstructed BDs, using the Levenshtein distance as a measure. We demonstrated that the results of the evaluation method agree with those from qualitative comparison. In addition, we showed that the more time-series data sets we used, the more accurately the BD was reconstructed. The results show that the proposed method is useful for the quantitative evaluation of reconstructed 1-dimensional BDs.

In future work, we will demonstrate the efficiency of this evaluation method for 1-dimensional BDs of other dynamical systems. In addition, we will attempt to evaluate BDs that are reconstructed by using other time-series predictors, and to develop a time-series predictor whose reconstruction accuracy is higher than that of the ELM.

\section{References}

[1] Tokunaga, R., Kajiwara, S., \& Matsumoto, S. (1994). Reconstructing bifurcation diagrams only from time-waveforms. Physica, 79, 348-360.

[2] Ogawa, S., Ikeguchi, T., Matozaki, T., \& Aihara, K. (1996). Nonlinear modeling by radial basis function networks. IEICE Trans, E79-A (10), 1608-1117.

[3] Bagarinao, E., Pakdaman, K., Nomura, T., \& Sato, S. (1999). Reconstructing bifurcation diagrams from 
noisy time series using nonlinear autoregressive models. Physica Review E, 60, 1073-1076.

[4] Bagarinao, E., Pakdaman, K., Nomura, T., \& Sato, S. (1999). Time series-based bifurcation diagram reconstruction. Physica Review E, 130, 211-231.

[5] Bagarinao, E., Pakdaman, K., Nomura, T., \& Sato, S. (2000). Reconstructing bifurcation diagrams of dynamical systems using measured time series. Method Inform. Med, 39, 146-149.

[6] Langer, G., \& Parlitz, U. (2004). Modeling parameter dependence from time series. Physical Review E, 70, 1-9.

[7] Tada, Y., \& Adachi, M. (2013). Reconstruction of bifurcation diagrams using extreme learning machines. Proceedings of IEEE International Conference on Systems, Man, and Cybernetics (pp. 1127-1131).

[8] Itoh, Y., Tada, Y., \& Adachi, M. (2015). Reconstruction of bifurcation diagrams with lyapunov exponents for chaoric systems from only time-series data. Proceedings of 2015 International Symposium on Nonlinear Theory and its Applications (pp. 692-695).

[9] Shimada, I., \& Nagashima, T. (1979). A numerical approach to ergodic problem of dissipative dynamical systems. Prog. Theor. Phsy, 61 (6), 1605-1616.

[10] Sano, M., \& Sawada, Y. (1985). Measurement of the lyapunov spectrum from chaotic time series. Phys. Rev. Lett., 55, 1082-1085.

[11] Adachi, M., \& Kotani, M. (1979). Identification of chaotic dynamical systems with back-propagation neural networks. IEICE Trans. Fundamentals, E77-A(1), 324-334.

[12] Levenshtein, V. I. (1966). Binary codes capable of correcting deletions, insertions and reversals. Soviet physics doklady, 10, 707-710.

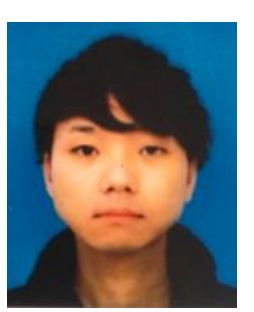

Yoshitaka Itoh is a doctoral student of graduate school of advanced science and technology, Tokyo Denki University, Japan. He received his master degree in engineering from Tokyo Denki University, Japan in 2011. He has worked at NEC informatec systems. His main research area is nonlinear time series prediction and analysis of chaotic system. He is a member of IEEE.

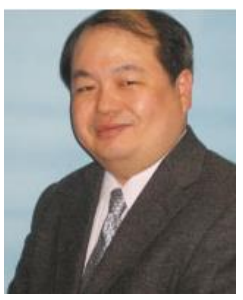

Masaharu Adachi is a professor of graduate school of advanced science and technology, Tokyo Denki University. His research interests include supervised and unsupervised learning for artificial neural networks, associative memory especially with chaotic dynamics and nonlinear time series prediction. He is a member of IEEE and IEICE. 